\title{
INFECCIÓN INTRAHOSPITALARIA
}

\section{Serratia marcescens: Descripción de un brote de infección intrahospitalaria}

\author{
M. TERESA DOSSI C. ${ }^{1}$, MARCELA ESCALONA U. ${ }^{1}$, CRISTIÁN SERRANO A. ${ }^{1}$, \\ M. ANGÉLICA SILVA D. ${ }^{2}$; CHRYSTAL JULIET L. ${ }^{2}$, ALEJANDRA FERNÁNDEZ V. ${ }^{2}$, \\ VERÓNICA LEIVA C. ${ }^{2}$ y JORGE FERNÁNDEZ O. ${ }^{3}$
}

\section{SERRATIA MARCESCENS: DESCRIPTION OF A NOSOCOMIAL OUTBREAK}

We describe a nosocomial outbreak of Serratia marcescens infections in different departaments, which occurred between May and December 2001. It involved 85 patients, 72 with clinical infections and 13 in which the strains isolated were found as colonization. The most common site was urinary tract infection. Thirty six percent of the infections were severe (including pneumonia and bacteremia), occurring in patients older than 65 years, with predisposing conditions, previous antibiotic usage, prolonged hospitalisation and invasive procedures. Global lethality was 2,8\%. All the cases had genetically the same strain, so a common source of infection was thought. The environmental and water microbiological studies yielded no growth of Serratia, except for the water faucet in the Neurology ward, where the index case was detected, and probably where the epidemic originated.

Key words: Nosocomial infection; Serratia marcescens; Outbreak.

\section{INTRODUCCIÓN}

Serratia marcescens es un bacilo Gram negativo de la Familia Enterobacteriaceae que puede encontrarse en la flora intestinal del hombre y animales, en el ambiente y en reservorios pobres en nutrientes como el agua potable, cañerías y llaves, así como también en insumos hospitalarios como jabones, antisépticos, etc. $\mathrm{Su}$ adquisición es mayoritariamente nosocomial, especialmente en unidades de cuidados intensi$\operatorname{vos}^{1}$, siendo secreciones respiratorias, heridas y orina, sitios frecuentes de colonización ${ }^{2}$. Exis- ten reportes de brotes epidémicos de $S$. marcescens que señalan como potenciales fuentes de transmisión los equipos de ventilación mecánica, desinfectantes, jabones y manos ${ }^{3,4}$, otorgándose un rol fundamental en su origen al quiebre de la técnica aséptica, la reducción en la frecuencia en el lavado de manos y el incumplimiento de las normas destinadas al control de infecciones nosocomiales 5 .

Clínicamente, las bacteremias por S. marcescens se presentan con mayor frecuencia en pacientes con enfermedades de base como diabetes, neoplasias e insuficiencia renal crónica ${ }^{1,6}$.

\footnotetext{
Estudiante de Medicina, Facultad de Medicina, Universidad de Chile.

Unidad Microbiología y Epidemiología Hospital Del Salvador.

Sección de Desarrollo Tecnológico ISP.
} 
Las principales puertas de entrada de este microorganismo son la urinaria, respiratoria y vascular $^{1}$. La mortalidad asociada a este agente oscila entre 25 y $32 \%$.

La tasa de ataque de infecciones intrahospitalarias por $S$. marcescens en el Hospital Del Salvador, durante el año 2000 fue de $0 \%$ en un total de 31.012 egresos. Esta tasa se mantuvo hasta abril del 2001. El $1^{\circ}$ de mayo se aislaron en la UCI, dos cepas de S. marcescens con igual antibiotipo en dos pacientes, uno de los cuales provenía del Servicio de Neurología (caso índice). Pocos días después se volvió a aislar este mismo agente en 4 pacientes del Servicio de Medicina, iniciándose un brote epidémico que involucró 85 casos y que se extendió hasta diciembre. La tasa de ataque aumentó a 0,28\% en un total de 30.024 egresos el año 2001. Este brote motivó la búsqueda de las posibles fuentes y vías de transmisión, en reservorios descritos en la literatura ${ }^{4,7}$.

El objetivo de esta comunicación es describir las características epidemiológicas del brote epidémico por $S$. marcescens, la localización de las infecciones y la evolución clínica de los casos graves (neumonías y bacteremias).

\section{PACIENTES Y MÉTODO}

Se definió como caso a todo paciente hospitalizado con aislamiento de $S$. marcescens con antibiotipo característico (descrito en resultados), el cual en todos los casos correspondió al mismo genotipo. Se incluyeron por lo tanto colonizaciones e infecciones. Se definió como colonización al aislamiento de este agente en pacientes sin manifestaciones clínicas ni de laboratorio de enfermedad, y en los cuales no se indicó tratamiento antimicrobiano. Como infección se consideró al aislamiento de esta especie a partir de pacientes con manifestaciones clínicas y de laboratorio de enfermedad, por lo cual recibieron tratamiento antimicrobiano específico.

El estudio consistió en la revisión de las historias clínicas de todos los casos involucrados en el brote mediante una pauta predefinida, y en la búsqueda microbiológica de las posibles fuentes de infección: antisépticos, vaselina lí- quida, envases plásticos, recipientes y utensilios utilizados en Farmacia. Además se tomaron muestras de los antisépticos en uso y grifos de lavamanos de las diferentes salas comprometidas. La obtención de estas últimas muestras se realizó introduciendo una tórula en el interior del grifo. Para el estudio microbiológico se sembraron las muestras líquidas en caldo tripticasasoya, se incubaron a $35^{\circ} \mathrm{C}$ durante 72 horas y posteriormente fueron resembradas a agar sangre y McConkey. Las muestras tomadas con tórula (humedecidas previamente en caldo tripticasa) se sembraron directamente en agar sangre y McConkey. La identificación de las cepas se realizó de acuerdo a técnicas standard de laboratorio (Murray, 1999) y fue confirmada por la Sección Enterobacteriaceas del ISP. En total se tomaron 40 muestras de estas posibles fuentes ambientales.

Para el estudio genético de clonalidad se enviaron al ISP las primeras 10 cepas aisladas del brote, una cepa obtenida de la investigación de las fuentes ambientales y además, dos cepas aisladas de pacientes ambulatorios, con diferente antibiotipo al de las cepas del brote.

Se solicitó al Servicio de Salud Metropolitano del Ambiente-SESMA, el estudio químico microbiológico de las aguas de los estanques que surtían los servicios afectados por este brote. Se excluyó de este estudio el estanque de aguas de uso exclusivo de los servicios de Obstetricia y Neonatología.

\section{RESULTADOS}

El brote se inició en mayo del 2001, siendo el caso índice un paciente que presentó una bacteremia de origen urinario en el Servicio de Neurología. La cepa de Serratia presentó un antibiotipo característico, siendo sensible a ciprofloxacina, cefepime, meropenem, imipenem; medianamente sensible a amikacina y sulperazona, y resistente a gentamicina, ampicilina/ sulbactam, cefalosporinas de $1^{\mathrm{a}}$ y $2^{\mathrm{a}}$ generación y ceftriaxona. Los aislamientos se extendieron hasta diciembre del mismo año, comprometiendo otros servicios clínicos e involucrando un total de 85 pacientes, de los cuales 72 fueron infecciones y 13 colonizaciones. 
La curva epidémica del brote se observa en el Gráfico 1.

Los servicios clínicos que registraron mayor cantidad de aislamientos fueron: SAIC (sala de cuidados intermedios quirúrgicos), UCI y SAIM (sala de tratamiento intermedio de Medicina). Se destaca la ausencia de casos en los servicios de Obstetricia y Neonatología. El Gráfico 2 indica tasa de ataque expresada en porcentaje según egresos por servicio clínico. En el Gráfico 3 se observa la tasa de ataque por servicio clínico en el tiempo.

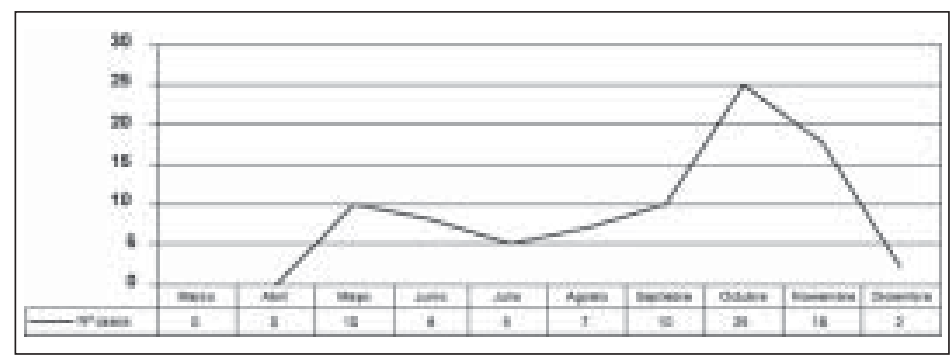

Gráfico 1. Curva epidémica de marzo a diciembre 2000.

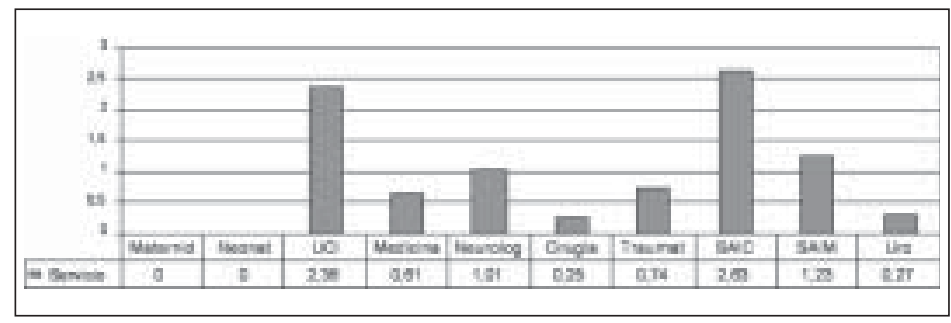

Gráfico 2. Tasa de ataque por egresos según servicio clínico

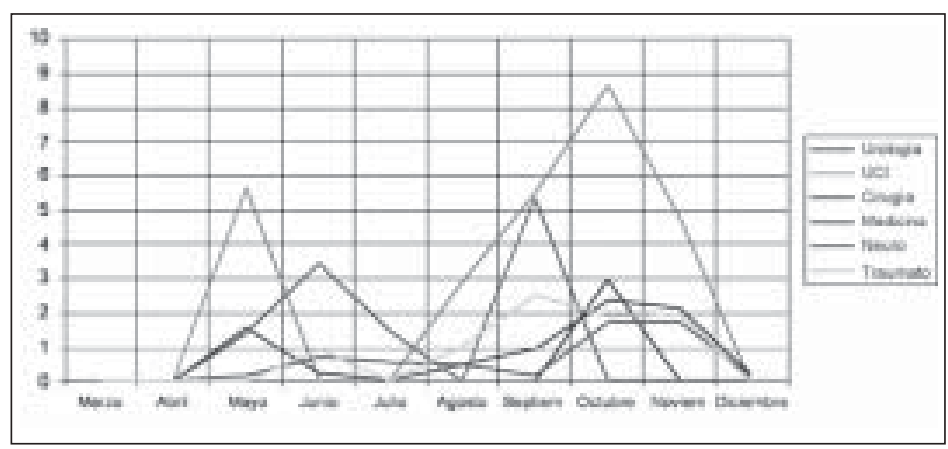

Gráfico 3. Tasa de ataque por servicio clínico en el tiempo

\section{Caracterización clínica de los casos:}

Serratia marcescens afectó a 85 pacientes provocando 13 colonizaciones y 72 infecciones. La localización de las infecciones se detalla en la Tabla 1.

El 36\% (26) de las infecciones fue grave, 16 correspondieron a hombres y 10 a mujeres, siendo el promedio de edad 64,6 años (54\% mayor de 65). Las localizaciones fueron neumonías 11 y bacteremias 15. De éstas, 6 se asociaron a CVC, 5 a foco urinario y 4 fueron de aparición postquirúrgica. Entre los factores predisponentes destacaron: patología de base en $85 \%$ (neoplasias 7, alcoholismo 3, diabetes 3 , desnutrición 3 , insuficiencia cardiaca 3 , insuficiencia renal crónica 3); antimicrobianos previos en $77 \%$ (20); hospitalización prolongada en todos los pacientes (mayor a 30 días en promedio); cirugía previa en 65 $\%$ (17) y procedimientos invasores en $77 \%$ (CVC 18, cateterismo vesical 20 , ventilación mecánica 5). El $77 \%$ se trató con ciprofloxacina teniendo una evolución satisfactoria. Fallecieron 2 de los 26 casos con infecciones graves, uno por neumonía, y otro por bacteremia de foco urinario, siendo la causa de la muerte atribuible a la infección por este agente. Estos últimos pacientes fueron tratados con esquema antimicrobiano empírico que no incluyó quinolonas.

\section{Estudio microbiológico del brote}

Los cultivos realizados en las 40 muestras de insumos de Farmacia y salas de los servicios clínicos fueron negativos para S. marcescens, a excepción de un grifo del lavamanos de la sala de Neurología donde se detectó el primer caso.

El estudio químico microbiológico realizado por el SESMA 
Tabla 1. Brote de infección nosocomial por Serratia marcescens

\begin{tabular}{lcc}
\hline Localización de las infecciones & $\mathbf{N}^{\circ}$ & $\boldsymbol{\%}$ \\
\hline Infección urinaria & 27 & 37,5 \\
Infección herida & 19 & 26,8 \\
Neumonía & 11 & 15,2 \\
Bacteremia asociada a catéter venoso central & 6 & 8 \\
Bacteremia de foco urinario & 5 & 7 \\
Bacteremia postquirúrgica (foco abdominal) & 4 & 5,5 \\
Total & 72 & 100 \\
\hline
\end{tabular}

a las aguas de los estanques que surtían los servicios clínicos comprometidos en el brote no detectó $S$. marcescens y reveló niveles aceptables de cloración y desarrollo microbiano.

\section{Estudio genético del brote}

El estudio genético de análisis de los patrones de ácido nucleico mediante electroforesis en gel de campo pulsado realizado en el ISP, mostró que las primeras 10 cepas del brote, correspondientes a infecciones, y la cepa aislada del grifo de la sala de Neurología, tenían el mismo genotipo. Las 2 cepas de $S$. marcescens aisladas de pacientes ambulatorios con otro antibiotipo, revelaron genotipos diferentes a las cepas del brote y entre sí.

Las medidas de intervención se iniciaron al diagnosticarse los primeros 6 casos en el mes de mayo. En Farmacia, después de evaluados los procesos de preparación y despacho de desinfectantes, se decidió la compra de estos en envases desechables individuales de poco volumen para cada sala, eliminando el envío de ellos en recipientes reciclables de mayor volumen. En los otros servicios comprometidos se extremaron las medidas efectivas de control de infecciones y supervisión de la técnica aséptica al personal de enfermería en la realización de procedimientos invasores (instalación de catéter vesical, aspiración de secreciones, curación de heridas) y en el estamento médico, la instalación de los catéteres endovenosos. En las unidades críticas y en los servicios de Medicina y Cirugía se realizaron reuniones clínicas de capacitación a los médicos informando sobre el brote y la importancia del cumplimiento de las normas del Comité de Infecciones Intrahospitalarias.

\section{COMENTARIO}

Se describe un brote de IIH causado por $S$. marcescens, constituido por 72 infecciones ( $36 \%$ severas) y 13 colonizaciones, que afectó especialmente a las unidades críticas del hospital, tanto médicas como quirúrgicas. Desde el punto vista clínico, este brote comprometió a pacientes sobre 60 años de edad, siendo los principales factores predisponentes cirugía previa, procedimientos invasores, uso de antimicrobianos previos y hospitalización prolongada. La mortalidad global de las infecciones fue de $2,8 \%$, siendo $8 \%$ para las infecciones graves, la cual es muy inferior a la comunicada en la literatura $(25-30 \%)$.

Todos los casos correspondieron genéticamente a la misma cepa por lo que se planteó una probable fuente común de infección. Sólo uno de los 40 cultivos del ambiente tomados en busca de la fuente, que correspondió a una llave del lavamanos de la sala del caso índice (Servicio de Neurología) resultó ser positivo a $S$. marcescens coincidiendo su genotipo con el de las cepas causantes del brote. Del comportamiento de la curva epidémica (larga duración, con algunos picos en el tiempo), del estudio microbiológico y genético, se deduce la hipótesis de que el brote se originó desde esta fuente común y se diseminó al resto de los servicios vía mano portada, manteniéndose el reservorio en los pacientes infectados o colonizados. Esta hipótesis se corroboraría con el término del brote al insistir en el cumplimiento de las normas de IIH en los diferentes servicios.

\section{RESUMEN}

Se describe un brote de infección intrahospitalaria por Serratia marcescens ocurrido entre mayo y diciembre del 2001 en el Hospital Del Salvador, comprometiendo a varios servicios clínicos. Este brote involucró 85 pacientes con 72 infecciones y 13 colonizaciones. La 
localización más frecuente de las infecciones fue la urinaria. El $36 \%$ de las infecciones fue grave (neumonías y bacteremias), afectando a pacientes sobre 65 años de edad, con patologías predisponentes como: hospitalización prolongada, procedimientos invasores, cirugía y uso de antimicrobianos previos. La letalidad global fue de $2,8 \%$. Todos los casos correspondieron genéticamente a la misma cepa por lo que se planteó una probable fuente común de infección. El estudio microbiológico de las muestras ambientales y de las aguas no detectó $S$. marcescens, a excepción de un grifo del lavamanos del Servicio de Neurología donde se detectó el caso índice, por lo cual se puede deducir que probablemente éste fue el origen del brote epidémico.

\section{BIBLIOGRAFÍA}

1.- HADDY R I, MANN B L, NADKAMI D D, CRUZ R F, ELSHOFF D J, BUENDIA F C, DOMERS T A, OBERHEU A M. Nosocomial infections in the community hospital: severe infection due to Serratia species. J Fam Pract 1996; 42 (3): 273-7.

2.- Demetriou CA, Cunha BA. Serratia marcescens bacteremia after carotid endarterectomy and coronary artery bypass grafting. Heart Lung 1999; 28 (4): 293.

3. - ONES B L, GORMAN L J, SIMPSON J, CURRAN E T, MC NAMEE S, LUCAS C, MICHIE J, PLATT D J, THAKKER B. An outbreak of Serratia marcescens in two neonatal intensive care units. $\mathrm{J}$ Hosp Infect 2000; 46(4): 314-9.

4.- Nosocomial Serratia marcescens infections associated with extrinsic contamination of a liquid nonmedicated soap. Infect Control Hosp Epidemiol 2000; 21 (3): 196-9.

5.- MANNING M L, ARCHIBALD L K, BELL L M, BANERJEE S N, JARVIS W R. Serratia marcescens transmission in a pediatric intensive care unit: a multifactorial ocurrence. Am J Infect Control 2001; 29(2): 115-9

6.- YU WL, LIN CW, WANG DY. Serratia marcescens bacteremia: clinical features and antimicrobial susceptibilities of the isolates. J Microbiol Inmunol Infect 1998; 31(3):171-9

7.- WENDTT C, HERWALDT L. Epidemics: Identification and management. En Prevention and Control of Nosocomial Infections. Wenzel R. Ed. Third edition .1997. Williams \& Wilkins. pp 190-2.

Correspondencia a:

Alejandra Fernández Valdés

E-mail: faraya1@mi-mail.cl 